\title{
Homo capax Dei. La posibilidad del conocimiento teórico de Dios según Leonardo Polo
}

Homo capax Dei. The Possibility of the Theoretical Knowledge of Cod According to Leonardo Polo

\section{Miguel Martí SÁnCHeZ}

Universidad de Navarra mmarti.1@aluni.unav.es
RECIBIDO: ? DE ? DE 201?

VERSIÓN DEFINITIVA: ? DE ? DE 201? DOI: $10.15581 / 013.19 .27-40$
Resumen: El problema del conocimiento teórico de Dios ha ocupado a los filósofos desde los orígenes de la Filosofía. El giro copernicano que Kant imprimió al planteamiento metafísico por excelencia -Dios- no hizo sino agudizar el asunto. Las múltiples tradiciones filosóficas contemporáneas también manifiestan un interés por la posibilidad o imposibilidad de tal empresa. Aunque la propuesta filosófica de Leonardo Polo no se inserta directamente en alguna de esas tradiciones, se aprovecha de muchas de sus nociones y argumentaciones, sobre todo de las tradiciones aristotélica, tomista y fenomenológica. Según el filósofo madrileño la inteligencia, también en su vertiente teórica, es capax Dei. El siguiente artículo pretende hacerse cargo de manera global de en qué consiste para Leonardo Polo ese aserto.

Palabras clave: Dios, inteligencia, método, límite, hábito, objeto, término, operación intelectual.
Abstract: The inquiry about the existence and essence of God has been a philosophical question since the origins of Philosophy. Kant's Kritik der reinen Vernunft gave a Copernican Turn to this issue; according to him, we cannot demonstrate the existence of God by means of the theoretical reason. In contemporary philosophy this question about God has been, by different traditions, revisited. The philosophical proposal of L. Polo -especially his contribution in theory of knowledgecould help to reconsider this topic with new tools and arguments. According to Polo the human intellect (inteligencia) in his theoretical dimension is essentially capax Dei. This article tries to summarize the arguments of Polo in order to support his thesis about the possibility of theoretical knowledge of God.

Keywords: Intellect, God, Method, Limit, Habit, Object, Culmination, Mental Act. 


\section{INTRODUCCIÓN}

juicio de Kant la razón teórica está esencialmente incapacitada para de-
mostrar de manera concluyente la existencia de un ser supremo. En la medi-
da en que:

"Un conocimiento teórico es especulativo cuando se refiere a un objeto o a conceptos de un objeto que no pueden ser alcanzados en ninguna experiencia. A él se opone el conocimiento de la naturaleza, que no se refiere a otros objetos o predicados de objetos que a los susceptibles de darse en una experiencia posible".

Por consiguiente no cabe apelar a meros conceptos si se quiere demostrar la realidad objetiva (objektive Realität) de algo. En este sentido la experiencia posible (mögliche Erfahrung) es el único punto de partida lícito para un conocimiento (Erkenntnis) en sentido estricto. Ahora bien, Dios no es objeto de tal experiencia posible, ni como causa primera de la totalidad del mundo ni como fin al que tiende todo el cosmos. Con otras palabras, no se trata de que Kant critique las pruebas o demostraciones de la existencia de Dios -algo que también hace en la $\mathrm{KrV}$-, sino que según él tal intento está condenado de raíz al fracaso, tome la forma argumental que tome. De ahí que afirme:

"Sostengo, pues, que todas las tentativas de una razón meramente especulativa en relación con la teología son enteramente estériles y, consideradas desde su índole interna, nulas y vacías; que los principios de su uso natural no conducen a ninguna teología; que, consiguientemente, de no basarnos en principios morales o servirnos de ellos como guía, no puede haber teología racional ninguna, ya que todos los principios sintéticos del entendimiento son de uso inmanente, mientras que el conocer un ser supremo requiere hacer de ellos un uso trascendente para el que nuestro entendimiento no está equipado”².

${ }^{1}$ I. KANT, Crítica de la razón pura, Taurus, Madrid, 2005, Traducción de Pedro Ribas, 526: "Eine theoretische Erkenntnis ist spekulativ, wenn sie auf einen Gegenstand, oder solche Begriffe von einem Gegenstande, geht, wozu man in keiner Erfahrung gelangen kann. Sie wird der Naturerkenntnis entgegengesetzt, welche auf keine andere Gegenstände oder Prädikate derselben geht, als die in einer möglichen Erfahrung gegeben werden können" (Kritik der reinen Vernunft, Felix Meiner Verlag, 1998, A 635 / B 663).

2 I. KANT, Crítica de la razón pura, op. cit., 527: "Ich behaupte nun, daß alle Versuche eines bloß spekulativen Gebrauchs der Vernunft in Ansehung der Theologie gänzlich fruchtlos und ihrer inneren Beschaffenheit nach null und nichtig sind; daß aber die Prinzipien ihres Naturgebrauchs 
A su juicio, Dios siempre queda más allá de las posibilidades de la razón teórica, pues cuando trata de ser especulativa, es decir, cuando hace de sí misma un uso especulativo, no puede aspirar al mismo tipo de conocimiento que cuando se refiere al que está fundado en la experiencia posible que nos permite conocer la realidad natural. Por eso, según el regiomontano:

"El ser supremo se queda, pues, en mero ideal del uso meramente especulativo de la razón, aunque sea un ideal perfecto, concepto que concluye y corona el conocimiento humano entero y cuya realidad objetiva no puede ser demostrada por este camino, pero tampoco refutada"3.

Ahora bien, ¿es ésta la única posibilidad de concebir la razón teórica, a saber: en su distinción entre uso especulativo y uso dentro de la experiencia posible? A mi juicio Leonardo Polo ofrece una investigación de la inteligencia que permite ir más allá de la distinción trazada por el filósofo regiomontano, y en ese caso, mostrarla como capaz esencialmente de Dios.

\section{LA POTENCIALIDAD INFINITA DE LA INTELIGENCIA}

La noción de 'inteligencia' ocupa un lugar principal en la especulación de Polo, una prueba de ello es su uso constante en su Curso de Teoría del Conocimiento. Una de las notas principales que Polo asigna a la inteligencia es que, en palabras suyas: "no tiene fondo de saco", es decir, que aunque tenga un límite, eso no significa que termine -en el sentido de acabar algo- con algún objeto último. El filósofo español suele servirse de la argumentación de san Anselmo y el argumento ontológico para criticar la visión de que cupiese la posibilidad de un objeto que agotase la capacidad cognoscitiva del entendimiento humano.

Ahora bien, esto no debe entenderse como si para Polo la inteligencia humana fuese una potencia infinita de concebir, de hecho procede mediante

\footnotetext{
ganz und gar auf keine Theologie führen, folglich, wenn man nicht moralische Gesetze zum Grunde legt, oder zum Leitfaden braucht, es überall keine Theologie der Vernunft geben könne. Denn alle synthetische Grundsätze des Verstandes sind von immanenten Gebrauch; zu der Erkenntnis eines höchsten Wesens aber wird ein transzendenter Gebrauch derselben erfordert, wozu under Verstand gar nicht ausgerüstet ist" (KrV, op. cit., A 636/ B 664).

${ }^{3}$ I. KANT, Crítica de la razón pura, op. cit., 530: "Das höchste Wesen bleibt also für den bloß spekulativen Gebrauch der Vernunft ein bloßes, aber doch fehlerfreies Ideal, ein Begriff, welcher die ganze menschliche Erkenntnis schließt und krönet, dessen objektive Realität auf diesem Wege zwar nicht bewiesen, aber auch nicht widerlegt werden kann" (KrV, op. cit., A 641/ B 669).
} 
un conocimiento que podría denominarse aspectual, ya sea en forma de conocimiento por objetos o mediante hábitos, pero sí en cuanto que es infinita según la potencia. Puede ser útil para comprender el significado que Polo asigna aquí a la noción de 'infinito en potencia' acudir a la definición que hace de él Aristóteles en el libro III de la Física ${ }^{4}$. Allí dice el Estagirita:

"Pues bien, 'ser' se dice, por un lado 'ser en potencia' y por otro 'ser

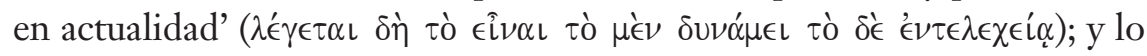

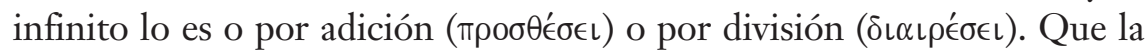

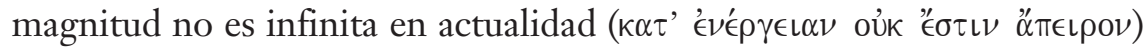

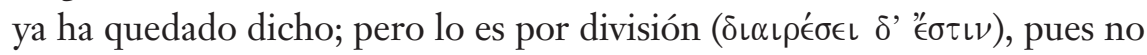
es difícil refutar las líneas indivisibles. Nos resta, pues, que lo infinito sea

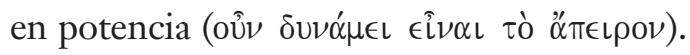

Pero no hay que tomar 'lo-que-es-en-potencia' en el sentido en que, si es posible que esto sea una estatua esto será una estatua, así también es infinito lo que va a serlo en actualidad. Antes bien, dado que 'ser' se dice

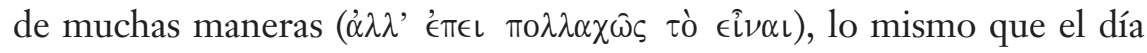
'es' y la competición 'es' por el hecho de producirse sucesivamente un su-

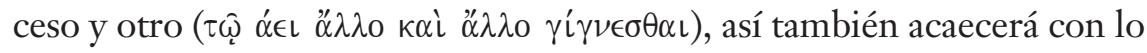
infinito. (Claro que, en el caso de aquéllos, 'son en potencia y en actualidad': los juegos Olímpicos 'son' tanto porque la competición pueda tener lugar, como por el hecho de que tiene lugar).

En cambio, lo infinito se entiende evidentemente de forma diferente $(\alpha \lambda \lambda \omega \varsigma)$ en el caso del tiempo y de los hombres, que en el caso de la división de las magnitudes. En general, lo infinito es así por el hecho de to-

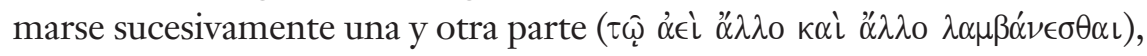
y por el hecho de que lo que se toma siempre es finito, aunque siempre,

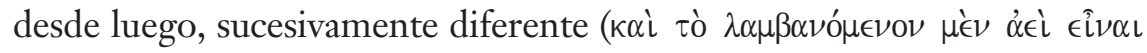

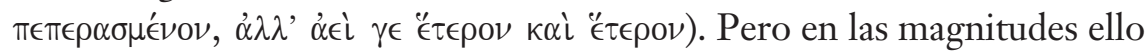

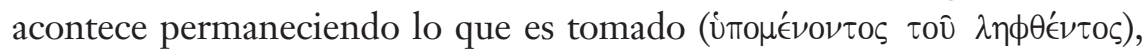
mientras que en el caso del tiempo y de los hombres, que van perecien-

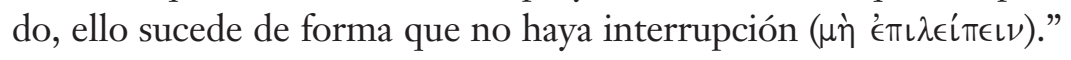

Interesa quedarse de este texto sobre todo con la idea de que nunca es posible la actualidad completa de lo que es infinito, es decir, que sea infinito significa

${ }^{4}$ Cfr. Fiss., III 6, 206 a 15-203 b 2 (ARISTÓTELES, Física [texto y traducción de José Luis Calvo Martínez], CSIC, Madrid, 1996). 
que siempre queda un resto sin actualizar que es justo el signo de que algo es potencialmente infinito. A mi juicio, éste es el sentido que Polo asigna al término 'infinitud' cuando lo aplica a la inteligencia humana, a saber: nunca actualiza plenamente su capacidad de comprehender la realidad en todas sus formas, sino que se desenvuelve progresivamente. De ahí que el filósofo madrileño diga que no cabe un último objeto para la inteligencia humana, pues eso significaría también el término -casi "muerte"- de la misma inteligencia. Ocurre, más bien, lo contrario, la inteligencia nunca actualiza totalmente sus capacidades, y menos todavía con algún objeto.

\section{LA RADICACIÓN DE LA INTELIGENCIA EN LA PRINCIPIALIDAD}

La inteligencia no tiene un objeto último, pero eso no significa que se desenvuelva en el vacío. Más bien, según Polo, que no quepa un objeto definitivo o completo de la inteligencia tiene que ver con la apertura irrestricta de la inteligencia a la principialidad ${ }^{5}$. Este concepto debe entenderse en el sentido de lo que es último en sentido de prius con respecto a la capacidad de inteligir, es decir, es aquello con lo que se encuentra la inteligencia cuando comienza su actividad . Polo habla en este caso del 'ser' como principio trascendental, de una manera que recuerda al epigrama tomista de que "lo primero que cae en el entendimiento es el ente", pero sin una asimilación completa7.

Que los principios en sentido estricto son primeros con respecto a la inteligencia no significa que la inteligencia se deduzca o proceda de los principios, pues en cierto sentido, ella misma es como cierto principio, en la medida en que su actividad es irreductible a la de los principios "reales". Una frase de Polo en el artículo Lo intelectual y lo inteligible lo ilustra bien y de modo sintético: "reducido el entender a ser no es entender alguno: sólo ser sin entender que lo entienda. Y si ser se reduce a entender, sólo entender que no entiende nada"

\footnotetext{
5 Sobre este término puede verse el artículo de Polo: "Dios y la infinitud de la intelección”, en Studia Poliana, 14 (2012), 13-20.

${ }^{6}$ Sobre los sentidos de 'comenzar' en la filosofía de Leonardo Polo véanse los artículos de F. HAYA, "La superación del tiempo (I): la discusión de Polo con Husserl y Heidegger", en Studia Poliana, 5 (2003), 75-102; "La superación del tiempo (II): el estatuto de las modalidades según Polo", en Studia Poliana, 7 (2005), 41-74; "La superación del tiempo (III): los sentidos del comienzo", en Studia Poliana, 8 (2006), 151-181.

7 Véase su crítica a Descartes y su olvido de la actividad propia de la inteligencia y su radicación originaria en el ser en su libro Evidencia y realidad en Descartes.

${ }^{8}$ L. POLO, "Lo intelectual y lo inteligible", en Anuario Filosófico, XV/2 (1982), 110.
} 
Sin esa radicación primigenia de la inteligencia en la principialidad ésta no podría "ponerse en marcha". Aunque lo que Polo entiende por 'ser" en ese texto no se reduzca a la condición de posibilidad del conocimiento intelectual -es mucho más que eso- puede ser descrito, sin malinterpretar su sentido, como tal condición. De hecho así se aporta una nueva perspectiva de en qué sentido se puede hablar de 'ser' aparte de la 'intelección' -y viceversa-, pues se tiene en cuenta que la 'intelección' o 'entender' ya desde siempre están frente y en relación con 'lo que es'. En la medida en que se reconoce la radicación de la inteligencia en la principialidad se está en una situación idónea para describir lo propio de cada uno de ellos ${ }^{10}$.

\section{LA NOCIÓN DE HÁBITO INTELECTUAL}

Para Polo la inteligencia no es una capacidad cognoscitiva estática y monolítica sino dinámica y plural. Junto a la capacidad de captar objetos determinados -abstractos, conceptos universales, ideas generales, etc.- puede también remontarse más allá de ellos, sin por ello, abandonar el ámbito o esfera de los inteligibles. Su método del 'abandono del límite mental' no implica una deriva hacia lo irracional o lo místico -como podría parecer a una visión superficial de su propuesta filosófica- sino cierto reconocimiento de la potencialidad de la inteligencia. Que el intelecto humano no tiene "fondo de saco" significa que una vez se intelige es siempre capaz de más intelecciones y que ese tipo de conocimiento, por tanto, no es homogéneo sino que se diferencia según cierto orden; por ejemplo, entre la operación que conoce y el objeto conocido.

La distinción que para Polo juega un papel crucial es la que se da entre actos que conocen objetos -operaciones- y actos que conocen actos -hábitos-. Es útil repetir que la noción de 'hábito' en el ámbito intelectual no tiene sin más el mismo significado que en el terreno de la voluntad o del carácter. Ya Aristóteles distinguía entre virtudes morales o éticas y virtudes y hábitos intelectuales o dianoéticos. La aportación del filósofo madrileño tiene que ver con una caracterización rigurosa y peculiar de la fisionomía de los hábitos de la

\footnotetext{
9 Acerca de este término en la tradición tomista puede consultarse el libro de Á. L. GONZÁLEZ, Ser y participación, Eunsa, Pamplona, ${ }^{32001 ;}$ y, con matices pues no mantienen exactamente la misma tesis, el libro de E. GILSON, El ser y los filósofos, Eunsa, Pamplona, ${ }^{5} 2009$.

${ }^{10}$ Como se decía, estas ideas las desarrolla Polo sumariamente en el artículo "Lo intelectual y lo inteligible", en Anuario Filosófico, XV/2 (1982), 103-132.
} 
inteligencia ${ }^{11}$. Según él no se trata sólo de habilidades o competencias -como podría ser la astucia o la perspicacia intelectual-, sino más bien de que la inteligencia queda siempre detrás de sus operaciones a la manera de una cascada, es decir, que su núcleo no aparece sin más en alguna de sus operaciones. Advertir esta prioridad óntica de la inteligencia con respecto a sus manifestaciones abre el camino a una consideración no meramente 'operacionalista' del intelecto. De ahí que Polo diga que la pluralidad de operaciones intelectuales es solidaria con la pluralidad de hábitos.

Como afirma el filósofo madrileño:

"Sin embargo, los hábitos de la inteligencia son muy distintos de los de la voluntad. La primera razón por la que se consideran como perfecciones constitutivas de la potencia es, como he dicho, su asimilación a los hábitos de la voluntad. Pero esta asimilación no es correcta, porque los hábitos de la inteligencia no se adquieren por una repetición de operaciones, sino por una sola. Ésta es una tesis muy audaz, pero enteramente segura para Tomás de Aquino. El que ha ejercido una operación matemática ya tiene el hábito matemático, el conocimiento habitual de la matemática. Ahora bien, si esto es así, la asimilación aludida se ha de declarar imposible. No es lo mismo un hábito que se adquiere de una sola vez y no es incrementable, que un hábito que se adquiere con una repetición de actos y nunca acaba de poseerse plenamente. La inteligencia no es la voluntad, y su modo de adquirir hábitos es distinto. La diferencia primera es que a la inteligencia le basta un solo acto para adquirir un hábito y a la voluntad no, sino que necesita una pluralidad de actos, y nunca acaba de adquirirlos por completo" ${ }^{\prime 2}$.

Un hábito intelectual, dice Polo, se adquiere con sólo acto de la inteligencia, es decir, no por la repetición, sino por la consecución ${ }^{13}$. Además no debe equipararse con el ejercicio consciente y voluntario de una acción en el sen-

${ }^{11}$ Suele apoyarse en la cibernética para explicar este asunto. Cfr., entre otros, L. POLO, "La cibernética como lógica de la vida", en Studia Poliana, 4 (2002), 3-15.

12 L. POLO, Obras Completas XIV. Nominalismo, idealismo y realismo, Eunsa, Pamplona, 2016, 164.

13 "La noción de hábito es mucho más rica de lo que se suele decir: no es sólo una memoria intelectual, ni únicamente la perfección de la facultad, sino una iluminación de la operación; dicha iluminación culmina, digámoslo así, en un hábito que excluye la suficiencia del conocimiento objetivo, y de esa manera tematiza los primeros principios exclusivamente como primeros", L. POLO, Obras Completas V: Curso de Teoría del Conocimiento II, Eunsa, Pamplona, 2016, 233. 
tido de algo transeúnte y que persigue un fin externo a ella misma, sino que tiene que ver con el despliegue trascendental de la inteligencia, como si ésta contuviese in nuce lo que puede llegar a ser. No sorprende, entonces, que se diga que la inteligencia es susceptible de crecimiento irrestricto, siempre y cuando se entienda este crecimiento no como un desarrollo unilateral y uniforme, sino como un despliegue para la mejor comprensión de la realidad en sus distintos 'seres'.

La dinámica del hábito es la de un acto intelectual que reconoce la efectividad de la operación mental, y en esa medida garantiza la prosecución de la inteligencia al mantener la diferencia entre lo conocido y que eso ha sido conocido. La progresiva diferenciación permite la continua intelección sin perder de vista que la inteligencia siempre queda detrás -a modo de núcleo activo- del "estar inteligiendo".

\section{EL CRECIMIENTO IRRESTRICTO DE LA INTELIGENCIA}

La inteligencia o intelecto como capacidad dinámica y plural es susceptible de desarrollo y perfeccionamiento. No debe entenderse esto como si el télos de la inteligencia tuviese término, sino en el sentido de que es capaz de una progresiva actualización. Ahora bien, tan sólo porque ya está radicada en la principialidad nunca se agota, aunque esto no implica que encuentra su objeto frente a ella, en el sentido de algo que la "Ilenase" por completo. Más bien, por ser una capacidad esencialmente abierta de un modo peculiar ${ }^{14}$, no se sacia mediante un objeto, sino que está constantemente referida más allá de sí misma. A esto, aunque dicho de manera algo abrupta, es lo que Polo llama la 'carencia de réplica' del intelecto humano, pues no encuentra su último sentido en sí mismo o en otro ser sino más allá de $s i^{15}$.

Como explica Polo recurriendo a la noción de 'hábito', la potencia intelectiva no deja de perfeccionarse, porque:

"La inteligencia es operativamente infinita, pero no espontáneamente operativa (la espontaneidad [en el conocimiento] es una noción in-

${ }^{14}$ Polo suele servirse de la cita aristotélica en el De Anima: "el alma es en cierto sentido todas las cosas", en $D A$ III 8, $431 \mathrm{~b} 21$, para ilustrar su propuesta.

${ }_{15}$ No puedo desarrollar estas ideas por extenso, para el que quiera profundizar remito al excelente libro de F. HAYA, El ser personal. De Tomás de Aquino a la metafísica del don, Eunsa, Pamplona, 1997. 
sostenible), pues la operación no la perfecciona en cuanto que potencia: la perfección de una potencia formal debe ser, al menos formal, pero la operación no es una forma en acto. Paralelamente, la perfección de la inteligencia es habitual. He aquí una primera insuficiencia de la operación: es un acto que no perfecciona a la potencia, no es un hábito. Tal insuficiencia es externa a la operación; su iluminación es precisamente el hábito. Señalemos enseguida que un hábito no es sólo un acto formal, sino más que formal. Lo llamaré acto esencial en atención a que la inteligencia es una facultad del alma humana, la cual es forma y esencia. Esto significa: el hábito perfecciona la inteligencia de un modo que no corresponde a la operación, es decir, justamente como potencia. El hábito no 'despotencializa', a la inteligencia, sino que refuerza su carácter de potencia. La inteligencia habitual no deja de ser una potencia, sino todo lo contrario: es más potencia con hábito que sin él. Ello significa, a su vez, que perfeccionada por el hábito, no es potencia formal, sino potencia esencial. Ésta es estrictamente la primera insuficiencia de la operación intelectual y, en rigor, de cualquier operación intelectual: ninguna es hábito, ninguna es del orden de la esencia. El hábito es también una retracción, a saber, de la facultad como principio al principio de la facultad. Ese principio no es el alma como forma sustancial del cuerpo, sino el esse bominis, también el principio del alma como esencia. El intelecto agente no es el alma. Sin embargo, en tanto que ilumina la insuficiencia de la operación, tampoco el hábito es el acto de los inteligibles en acto" ${ }^{\text {16 }}$.

Algo semejante a la comprensión de la libertad que Polo realiza y que refiere a tres radicales distintos - griego, moderno y cristiano- ocurre con la inteligencia. Según el filósofo madrileño la libertad se ha entendido como dominio de uno mismo (griegos), como auto-realización y resultado (modernos) y como biperteleología y apertura a la trascendencia (cristianismo) ${ }^{17}$. Más allá de las posibles matizaciones que se podrían hacer a esta sumaria explicación, conviene notar que de hecho se enuncian tres modos fundamentales de entender la rela-

${ }^{16}$ L. POLO, Obras Completas V. Curso de Teoría del Conocimiento II, Eunsa, Pamplona, 2016, 247248.

${ }^{17}$ El desarrollo de este planteamiento lo hace L. Polo en "Lo radical y la libertad", cfr. Persona y libertad, Eunsa, Pamplona, 2007, 181-242; y en "Tener y dar", cfr. Obras Completas XIII. La persona humana y su crecimiento / La originalidad de la concepción cristiana de la existencia, Eunsa, Pamplona, 2015. 
ción entre el yo (o la persona) y el mundo circundante (o naturaleza en general). Y que ese modo de entender la relación cabe trasladarlo desde la esfera de la libertad a la del conocimiento intelectual. En pocas palabras: del mismo modo que la libertad en sentido moderno aboga por la búsqueda de su propia autorrealización a toda costa y como pura autonomía, por otro lado, tanto el radical griego como el cristiano se hacen cargo de la esencial incapacidad de tal empresa, y por eso sitúan al ser humano entre aquellos seres finitos cuya identidad les debe ser otorgada (ya sea a través de una comunidad, o bien de un ser superior, en principio ya idéntico a sí mismo); decíamos, del mismo modo, la inteligencia puede ser entendida, o bien como la capacidad del desvelamiento absoluto de la propia identidad y de la esencia del mundo, o bien como la capacidad de hacerse cargo progresiva pero infinitamente de lo otro y de esa manera remontarse a un principio último al que apelar ante la falta de respuesta mediante las propias fuerzas. A juicio de Polo sólo la segunda hace auténtica justicia a la dinámica propia de la inteligencia humana.

\section{LOS NIVELES DEL CONOCIMIENTO INTELECTUAL Y LA AUSENCIA DE TÉRMINO (PÉRAS)}

La operatividad y perfeccionamiento de la inteligencia, decíamos, no termina, en términos aristotélicos podría decirse que no tiene péras sino télos. Ahora bien, la imagen del proceso como algo continuo e interrumpible tampoco, piensa Polo, se hace cargo de la peculiar actividad que es el conocimiento intelectual. Su insistencia en el término 'enérgeia' -acuñado por Aristótelespara ejemplificar un determinado tipo de 'actos' o 'movimientos' debe interpretarse, a mi juicio, en este sentido; sin entrar en la mejor o peor hermenéutica que hace Polo de este término en la filosofía aristotélica.

Dicho télos de la inteligencia es lo que Polo denomina la principialidad y al tipo de mantenimiento en tal radicación lo llama también relación al Ori$g \mathrm{n}^{18}$. La terminología es peculiar pero manifiesta una intuición de fondo, a saber: inteligir -en sentido amplio, es decir, no restringido a las operaciones intelectuales sino en cuanto apertura trascendental a todos los entes, a sí mismo y a la principialidad- no es una actividad junto a otras manifestaciones humanas sino su punto focal. Esta apertura primigenia no debe verse como una tendencia hacia algo, en el sentido de una tensión ya establecida pero es extrínseca, sino más

\footnotetext{
${ }^{18}$ Cfr. su artículo: "La persona como relación al Origen”, en Studia Poliana, 14 (2012), 21-36.
} 
bien como un "estar enraizada" de tal manera la inteligencia en el 'principio' que la fuente de su actividad es también el fin de la misma. Esto sólo es posible por la insondabilidad e inagotabilidad del principio al que se encuentra "sujeto" el intelecto. Por esa razón, el "avance" de la inteligencia en su penetración no es en sentido propio y fundamental de tipo cuantitativo -saber más- sino cualitativo -saber mejor-: non multa sed multum.

Un texto de Evidencia y realidad en Descartes ilustra, a mi juicio, de manera clara y distinta esta idea de Polo:

"Sólo la realidad absoluta puede ser inteligida absolutamente. Esto significa: sólo la realidad absoluta es, indiscerniblemente, realidad e inteligible; sólo en la realidad absoluta la situación de objeto y la situación en $s i$, en cuanto determinadas por su distinción misma, son superadas y llevadas a coincidir en una situación unitaria más alta. La sustancia finita no es perfectamente inteligible y por lo tanto no puede ser en sí, directamente, ni siquiera objeto de conocimiento. Conocimiento exhaustivo de la realidad no significa para Descartes: conocimiento terminado desde el punto de vista, unilateral, de lo que hay que conocer, lo cual se encuentra plenamente desvelado y no guarda nada más. Esto no tiene sentido, porque olvida el conocimiento en cuanto tal. No cabe conocer perfectamente lo que no es perfecto, por cuanto la perfección del conocimiento no depende exclusivamente de lo conocido. La realidad en sí no puede ser conocida directamente en tanto que en sí: es decir, no puede ser directa e inmediatamente la realidad en sí porque lo conocido no puede consumarse terminativamente según la realidad finita. Una realidad conocida significa, a la vez: realidad-conocida $y$ conocimiento colmado, llevado a término. Esto último es necesario porque una realidad directamente conocida agota lo cognoscible, pues detrás de la realidad no hay nada. Si no hay nada más por conocer, el conocimiento llega a su término. Pero si la realidad no es el supremo cognoscible, la capacidad cognoscitiva no está saturada, y si no está saturada no está terminada. Llegamos así a probar que el conocimiento de la realidad finita exige en Descartes una actualidad situacional no real, la cual, a su vez exige superación. En el fondo, Descartes olvida la noción trascendental de ente por no interpretar el pensamiento como actividad. Es así como la finitud de la realidad se separa del desarrollo del anhelo de infinito y tiene que instrumentarse una situación simplemente distinta para la constitución del objeto en presencia para el 
sujeto. Al excluir toda potencialidad en la causa del conocimiento objetivo, Descartes lo reduce a insuperable finitud" ${ }^{\prime \prime}$.

\section{DiOS COMO TEMA DEL INTELECTO: LA IDENTIDAD ORIGINARIA}

Según Polo, a Dios no se llega con sucesivas operaciones intelectuales. La crítica de Polo al argumento ontológico de san Anselmo tiene como fin último mostrar esa imposibilidad. Según el filósofo madrileño dicha incompatibilidad se puede advertir cuando se detecta la esencial limitación del conocimiento operacional y objetivo. En la medida en que según la operatividad el objeto que se obtiene y con el cual se conmensura la operación es limitado, no cabe un objeto último ni diferente en su aparición al de cualquier otro objeto. En ese sentido, cabe decir, no sin cierto tono polémico, que para Leonardo Polo Dios no es, desde luego, objeto del conocimiento intelectual -entendido sólo como conocimiento de objetos $^{20}$ - Según él Dios si aparece es siempre como primigenio u originario, es decir, como aquello que precede y posibilita en sentido trascendental la actividad de cualquier intelecto bumano. De esta manera, según Polo, Dios sólo aparece tras haber abandonado el límite mental, y allí puede hacerlo, o bien como principio de identidad -en la primera dimensión del abandono-, o bien como Identidad Originaria -en la tercera dimensión de dicho método-.

Como explica el filósofo madrileño:

"La existencia de Dios se demuestra en la dirección de la advertencia de la actividad, la cual es solidaria del abandono del límite. La advertencia culmina en la noción de Incausado. La existencia de Dios es demostrada, pero no como término supuesto de la advertencia, sino en cuanto investida de todo el valor metódico de la primera dimensión del abandono del límite mental”21.

19 L. POLO, Obras Completas I. Evidencia y realidad en Descartes, Eunsa, Pamplona, 2015, 262.

${ }^{20}$ Véase la siguiente cita: "Por decirlo de algún modo, advertimos la inminencia, no la intimidad de la identidad originaria. Y ello porque la identidad no está contenida en la persistencia, simple referencia causal, y porque solamente con la referencia causal (creada) pueden advertirse las nociones de Incausado y de Origen. Dios es Incausado en tanto que la criatura es causa. Y esto no entraña necesitarismo alguno, porque Dios no es origen de la criatura" (L. POLO, El ser I: la existencia extramental, Eunsa, Pamplona, 2015, 211). La última afirmación que Dios no es origen de la criatura, no debe entenderse como que Dios no la cree, sino que Dios en tanto que Origen no precisa de la criatura para ser comprendido cabalmente; y por otro lado, que la criatura no emana de Dios, sino que se distinguen el uno de la otra radicalmente.

${ }^{21}$ L. POLO, El ser I, op. cit., 215. 
Y más adelante prosigue:

"La existencia de Dios no se puede demostrar a partir de la objetividad; de otro modo sólo se llega a la existencia empírica, y no hay tal existencia empírica de Dios"22.

En ninguno de los casos Dios se vuelve objeto del entendimiento humano, sino que más bien es descubierto o advertido como la antecedencia y prioridad irreductible a cualquier otro 'ser' o 'pensar'; por así decir, como la condición última de posibilidad en sentido radical.

De ahí que Polo afirme:

"Dios no existe en tanto que persiste. El acto de ser de Dios no es contradictorio porque no tiene nada que ver con el comenzar; es Increado. El principio de no contradicción es la intelección del acto de ser creado. El principio de identidad es la intelección del acto de ser increado. Pero si el acto de ser increado no está maclado con la no contradicción, tampoco lo está con la causalidad. Por decirlo de alguna manera, es exclusivamente identidad: puro principio de identidad distinto del puro principio de no contradicción”23.

Por esto el 'capax' de capax Dei no debe entenderse como un objetivo realizable para el entendimiento humano, sino más bien como el tipo de apertura y radicación de que precisa la inteligencia para desarrollar su actividad noética en todas sus facetas. En conclusión, si la inteligencia no se reduce a sus operaciones, sino que es superior a ellas y se refiere a Dios tanto como Identidad Originaria como Ser Incausado, entonces:

"Incausado significa tanto como Origen, es decir, como repulsa del ente en la plena simplicidad del ser, sin relación consigo como algo. Esta relación, por decirlo así, desaparece en la prioridad total. Dios no puede surgir a partir de Dios porque supera todo punto de partida. Dios no consiste en una contemplación de su esencia, ni en una afirmación voluntarista de su propia realidad. Dios es Origen, no resbala hacia ningún término; se posee tan activa y plenamente que la identidad, si vale la expresión, es absorbida, llevada culminariamente al Origen. Mientras

${ }^{22}$ Ibidem.

23 L. POLO, Nominalismo, op. cit., 221. 
la persistencia se refiere a la identidad, en Dios la identidad se realiza como un desbordamiento originario, como una expansión llevada al máximo originariamente y, por lo tanto, sin comienzo"24.

\section{Conclusión}

La razón teórica en su uso especulativo, dice Kant, va más allá de sus límites cuando pretende demostrar la realidad objetiva de un mero concepto, y tal cosa ocurre en el caso de un ser supremo, al que se suele denominar Dios. A juicio de Polo la posibilidad de demostrar la realidad o existencia de Dios a partir del conocimiento objetivo no está condenada al fracaso. Sin embargo, está en peor situación que una teoría de la inteligencia y del conocimiento que admita la pluralidad de operaciones y actos propios de la inteligencia. La teoría del conocimiento humano que reconoce la apertura del intelecto o inteligencia humanos a la principialidad, ya sea en forma de 'ser' como fundamento o de 'ser' como Origen, como decíamos, ofrece valiosas herramientas para elaborar una teología natural.

A mi juicio, la propuesta filosófica de Leonardo Polo ofrece valiosas indicaciones de cómo argumentar acerca de que caben demostraciones de la existencia de Dios, ya sea desde el conocimiento objetivo o habitual. Además, ofrece una visión de la inteligencia humana como esencialmente, o bien como solía decir Leonardo Polo "nativamente" capax Dei. Según él, un adecuado punto de partida para mostrar esta apertura radical de la inteligencia a Dios parece ser reconocer la peculiar forma de actividad que es propia de la inteligencia -enérgeia-, y que, por tanto, es irreductible al ente y a una ontología hecha sólo desde esa noción universalísima.

${ }^{24}$ L. POLO, El ser I, op. cit., 225. 